\title{
Personality and Problematic Smartphone Use: A Facet-Level Analysis using the Five Factor Model and HEXACO Frameworks
}

\author{
Sharon Horwood, Jeromy Anglim 1
}

\begin{abstract}
Problematic smartphone use can be defined as compulsive use that leads to impaired daily functioning in terms of productivity, social relationships, physical health, or emotional well-being. The current study provides a comprehensive assessment of how the broad and narrow traits of the HEXACO and Five Factor Models of personality predict problematic smartphone use. A sample of Australian adults ( $\mathrm{n}=393,79 \%$ female; mean age $=24.4, \mathrm{SD}=7.1$ ) completed the 300 -item IPIP NEO and the 200-item HEXACO-PI-R, along with measures of general and problematic smartphone use. Participants reported high levels of problematic smartphone use. Problematic smartphone use was positively correlated with neuroticism and negatively correlated with conscientiousness. Facet-level analysis highlighted the importance of several facets including impulsiveness, vulnerability, and anxiety as positive correlates and dutifulness, competence, self-discipline, and deliberation as negative correlates of problematic smartphone use. In the HEXACO framework, honesty-humility, agreeableness, conscientiousness, and openness all showed moderate negative correlations with problematic smartphone use, and emotionality was positively correlated with problematic smartphone use. Regression models indicated that narrow traits provide modest incremental prediction of problematic use. Overall, the study highlights the importance of personality traits for understanding predispositions to engage in problematic smartphone use.

Keywords: smartphones, problematic smartphone use, big five, hexaco, personality facets

\footnotetext{
${ }^{1}$ Horwood, S. \& Anglim, J. (2018). Personality and Problematic Smartphone Use: A Facet-Level Analysis using the Five Factor Model and HEXACO Frameworks. Computers in Human Behavior. https://doi.org/10.1016/j.chb.2018.04.013

Please see the doi to publisher's copy of record and check for updated volume and page numbers.

Sharon Horwood and Jeromy Anglim, School of Psychology, Deakin University, Geelong, Australia. School of Psychology, Deakin University, Geelong, Australia. Declarations of interest: none. Correspondence concerning this article should be addressed to Sharon Horwood, School of Psychology, Deakin University, Locked Bag 20000, Geelong, 3220 Australia. Email: sharon.horwood@ deakin.edu.au
} 


\section{Introduction}

Smartphones are a ubiquitous part of daily life for many people, and are transforming the ways that people interact, consume information, pass time, and get things done. Despite the many benefits of having continuous access to a multi-functional internet-enabled device, problematic smartphone use has been identified as an emerging public health issue (Billieux et al., 2015). We define problematic smartphone use as compulsive use that leads to impaired daily functioning in terms of productivity, social relationships, physical health, or emotional well-being. Although the causal direction is unclear, problematic smartphone use has shown links with a range of outcomes including negative mood (Collier, 2016; Elhai, Dvorak, Levine, \& Hall, 2017; Elhai, Levine, Dvorak, \& Hall, 2016; Matar Boumosleh \& Jaalouk, 2017; Yen, Ko, Yen, Wu, \& Yang, 2007), reduced physical fitness (Lepp, Barkley, Sanders, Rebold, \& Gates, 2013; Long et al., 2016), sleep deprivation (Christensen et al., 2016; Demirci, Akgönül, \& Akpinar, 2015; Lee, Lee, et al., 2014; Lemola, Perkinson-Gloor, Brand, Dewald-Kaufmann, \& Grob, 2015; Schweizer, Berchtold, Barrense-Dias, Akre, \& Suris, 2017), and poorer academic performance (David, Kim, Brickman, Ran, \& Curtis, 2014; Hawi \& Samaha, 2016; Tindell \& Bohlander, 2012; Wentworth \& Middleton, 2014). In addition to understanding how social norms and device features influence problematic use, researchers have begun to examine individual differences that predispose people to engage in problematic smartphone use.

Initial research on personality correlates of problematic smartphone use has principally focused on the Big 5 personality factors of extraversion, agreeableness, conscientiousness, neuroticism, and openness (Bianchi \& Phillips, 2005; Billieux, 2012; Butt \& Phillips, 2008; Ezoe, Toda, \& Yoshimura, 2009; Hussain, Griffiths, \& Sheffield, 2017; Kayiş et al., 2016; Kim et al., 2016; Lee, Chang, Lin, \& Cheng, 2014; Lepp, Li, Barkley, \& Salehi-Esfahani, 2015; Pearson \& Hussain, 2015; Phillips, Butt, \& Blaszczynski, 2006; Roberts, Pullig, \& Manolis, 2015; Shaw, Ellis, Kendrick, Ziegler, \& Wiseman, 2016; Stachl et al., 2017; Sween, Ceschi, Tommasi, Sartori, \& Weller, 2017; Takao, 2014; Takao, Takahashi, \& Kitamura, 2009; Toda, Ezoe, Mure, \& Takeshita, 2016). Previous Big 5 research suggests that higher neuroticism and lower conscientiousness are related to more problematic smartphone use. Despite the value of this initial research, several limitations can be noted. In particular, existing research has only focused on the Big 5, and has often relied on short-form, less reliable measures. A comprehensive understanding of the relationship between personality and any outcome is best achieved through the use of long-form comprehensive hierarchical measures 
of personality using large samples (Anglim \& Grant, 2014, 2016). Such an approach would allow for a comprehensive examination of how both broad and narrow traits predict problematic smartphone use. Thus, the present study sought to remedy this gap in the literature, by providing a comprehensive mapping of personality correlates of problematic smartphone use in relation to two of the most established hierarchical models of personality: the Five Factor Model (5 broad and 30 narrow traits; Costa \& McCrae, 2008 ) and the increasingly popular HEXACO Model (6 broad and 25 narrow traits; Lee \& Ashton, 2004).

\subsection{Problematic Smartphone Use}

The concept of problematic smartphone use is relatively new, and, as such, definitions are still being established. Some research defines problematic smartphone use broadly in terms of phone-related behaviors that negatively impact daily functioning (e.g. Elhai et al., 2017), while other researchers provide specific examples of problematic behaviors (e.g. not being able to go more than 1 hour without checking the phone, Hussain et al., 2017). Although some researchers prefer the term "problematic use" (Wang, Wang, Gaskin, \& Wang, 2015), others have adopted the clinical terminology of addiction (Kim et al., 2016; Y1ldı, 2017) with parallels to phenomena such as screen (Kardaras, 2016) and internet (Yen et al., 2007) addiction. Researchers have discussed how smartphone use has some of the general features of addiction, including dependence on the smartphone for pleasure, withdrawal symptoms if the smartphone becomes unavailable, cravings associated with wanting to be online and connected, and tolerance of excessive use (Hormes, Kearns, \& Timko, 2014; Y1ldı, 2017).

Various links between smartphone behavior and negative outcomes have been identified. For instance, many people use their smartphone after going to bed to check and respond to social media and messages (Deloitte, 2016a, 2016b), which has implications for sleep quantity and quality (Christensen et al., 2016; Lemola et al., 2015), and consequent health outcomes (e.g. depression, obesity, fatigue; Demirci et al., 2015; Elhai et al., 2017; Matar Boumosleh \& Jaalouk, 2017; Owens, 2014; Toda et al., 2016). Smartphone use can also impact on social and romantic relationship quality, with time spent on the device sometimes being a source of conflict (Deloitte, 2016a, 2016b). Furthermore, frequent use of smartphones while walking or driving can contribute to accidents. Such behaviors appear to be often characterized by mindless, habitual processes implying an inability to self-regulate smartphone use, while frequent attention switching and amount of time spent using smartphones have been identified as contributing to poorer academic performance in students (Hawi 
\& Samaha, 2016; Ishii, 2011; Kay, Benzimra, \& Li, 2017; Tindell \& Bohlander, 2012; Wentworth \& Middleton, 2014).

The relationships between mood disorders, reduced academic performance, and problematic smartphone use could be attributed to an emergent construct referred to as fear of missing out (FoMO, Elhai et al., 2016). Fear of missing out represents a desire to constantly stay online and connected via social media platforms, and has been established as a significant risk factor for the development of problematic smartphone use. When the desire to continuously interact online becomes excessive, behavioral indicators of addiction, similar to those attributed to drug and alcohol addiction, can emerge.

\subsection{Personality}

Personality traits can be conceptualized hierarchically, whereby broad factors are composed of, and defined by, several narrow facets. The most popular representation of broad traits is the Big 5. A particularly influential hierarchical representation of the Big 5 is the Five-Factor Model, where each of the Big 5 factors is composed of six facets (Costa \& McCrae, 1992). Despite the value of broad traits, research suggests that narrow traits provide incremental prediction and a more nuanced perspective on personality-criteria relationships (Anglim \& Grant, 2014; Paunonen \& Jackson, 2000). This finding has been shown for a range of outcomes including compulsive shopping (Otero-López \& Villardefrancos Pol, 2013), Type D Behavior (Horwood \& Anglim, 2017; Horwood, Anglim, \& Tooley, 2015), subjective well-being (Anglim \& Grant, 2016), job applicant faking (Anglim, Morse, De Vries, MacCann, \& Marty, 2017), academic performance (Anglim, Bozic, Little, \& Lievens, 2018), and values (Anglim, Knowles, Dunlop, \& Marty, 2017).

More recently, the six-factor HEXACO model of personality has emerged as an influential alternative to the Five Factor Model (Lee \& Ashton, 2004). The HEXACO model (an acronym for honesty-humility, emotionality, eXtraversion, agreeableness, conscientiousness, and openness) is composed of six broad factors that are each composed of four narrow facets, along with an interstitial facet of altruism. The HEXACO framework differs from the Big 5 in two important ways. First, the HEXACO factors of emotionality and agreeableness reflect a reorganization of the Big 5 factors of neuroticism and agreeableness (Ashton, Lee, \& de Vries, 2014). Second, the HEXACO model introduces a sixth factor, honesty-humility. The honest-humility factor measures positive traits such as sincerity, fairness, greed-avoidance, and modesty (Lee \& Ashton, 2004). While it shares some variance with FFM agreeableness, the honesty-humility factor is also strongly negatively correlated with 
the Dark Triad (Lee \& Ashton, 2005) and often incrementally predicts behaviors with a moral or transgressive quality (e.g., Dunlop, Lee, Ashton, Butcher, \& Dykstra, 2015; Lee, Ashton, Morrison, Cordery, \& Dunlop, 2008; Lee, Gizzarone, \& Ashton, 2003).

\subsection{Personality and Smartphone Use}

There is a growing interest in understanding how individual differences in general, and personality in particular, predispose certain people to engage in problematic smartphone use (Hussain et al., 2017; Kim et al., 2016; Roberts et al., 2015; Stachl et al., 2017; Sween et al., 2017; Takao, 2014; Takao et al., 2009; Toda et al., 2016). Despite research showing the importance of personality predicting problematic smartphone use, existing research has focused almost exclusively on the Big 5 (e.g. Kayiş et al., 2016; Roberts et al., 2015). Earlier studies on mobile phones rather than smartphones, found that several Big 5 factors predicted mobile phone usage. Extraverts typically spent more time using their phones overall, whereas those who were more neurotic, less agreeable and less conscientious spent more time sending text messages (Bianchi \& Phillips, 2005; Butt \& Phillips, 2008). More recently, various studies have implicated specific personality factors in the development of problematic smartphone use. For example, trait narcissism, neuroticism, and introversion have been linked to smartphone addiction (Pearson \& Hussain, 2015; Roberts et al., 2015).

Various theories for why personality predicts problematic smartphone use have been proposed. One theory is that individuals who are high in the traits of extraversion, approval motivation, and self-monitoring are more likely to spend excessive amounts of time using their smartphones to curate their online social profiles (Takao et al., 2009). The regular checking and updating of social media sites can lead to habitual use, and eventually excessive and problematic use (Hussain et al., 2017). This idea is supported by the finding that conscientiousness is negatively related to problematic smartphone use, whereby conscientious individuals are thought to be more self-disciplined and therefore less likely to habitually and frequently access their smartphones (Kayiş et al., 2016). Further possible explanations for the relationship between personality and smartphone use include neurobiological reinforcement via greater behavioral activation system rewards upon using a smartphone (Kim et al., 2016), and even the idea that excessive smartphone use can 'create' some personality traits in users (Pearson \& Hussain, 2015).

To date, personality and smartphone research has primarily relied on factor-level examinations. While one rigorous study examined correlations between objective smartphone use and 
personality at the factor- and facet-level (Stachl et al., 2017), the sample size of 137 was insufficient for a robust facet-level analysis (for a discussion of sample size requirements see Anglim \& Grant, 2014). Furthermore, the only two studies that have included HEXACO factors were primarily concerned with predicting a user's preferred operating system (i.e. Android or iOS; Shaw et al., 2016) or the likelihood of using a smartphone whilst driving (Sween et al., 2017). In summary, the lack a comprehensive mapping of extensive frameworks of personality on problematic smartphone use is a major gap in the literature.

\subsection{The Current Study}

Thus, the current study aimed to undertake an exploratory examination of the degree to which the Five Factor Model and the HEXACO model predict general and problematic smartphone use at both the factor and facet level. Various expectations can be derived from past research. First, given its links to a wide range of problematic behaviors, we expected neuroticism to be related to problematic smartphone use. Second, given its role in pursuing socially sanctioned goals and other health related behaviors, we expected conscientiousness to be associated with less problematic smartphone use.

\section{Method}

\subsection{Open Practices Statement}

Data, data analysis scripts, supplementary materials, and item-level information are provided at https://osf.io/nxv7z/

\subsection{Participants and Procedure}

The sample was drawn from a third-year undergraduate class on personality at an Australian university. Data collection took place in August 2017. Participants completed two online surveys that contained the personality inventories and other measures, and received their individual personality profiles in return. Given the length of the inventories, the provision of personality profiles to participants appeared to increase the motivation of participants to complete the questionnaire conscientiously. In the first session, participants completed demographics (age, gender, whether they used a mobile phone, and whether they used a smartphone), the 300-item IPIP personality test, the smartphone use measures, and some additional measures that were not used for the present study. In the second survey (completed on average 29.5 days after the first survey, $S D=9.9$ days), participants completed the HEXACO PI-R personality measure.

The final cleaned sample of smartphone users included 393 participants $(79 \%$ female; mean age $=24.4, S D=7.1$, $\min =18$, $\max =55$ ). This was based on an initial sample of 496, from which 
98 were removed due to not having completed all measures. Most of the removed cases $(n=90)$ did not complete the second survey. An examination of attrition showed that there was no significant difference in proportion female and mean age, Big 5 personality scores, and problematic smartphone use between participants who did and did not complete the second survey. Of the remaining 398 participants, five participants indicated that they did not use a smartphone, and one of these participants indicated that they did not use a mobile phone. These five participants were excluded from the final sample. Indicative of the saturation of smartphone use, $98.7 \%$ of the 398 participants used a smartphone.

\subsection{Materials}

\subsubsection{Problematic Smartphone Use}

The 27-item Mobile Phone Problem Use Scale (Bianchi \& Phillips, 2005) was used to measures problematic smartphone use. For the calculation of an overall problematic smartphone use variable, we excluded 8 items. Details for these exclusions are presented in the preliminary section of the results. In contrast to the original scale that used a 10-point scale from $1=$ not true at all to $10=$ extremely true, in order to reduce noise related to individual differences in response scale anchoring, we adopted a 5-point scale where $1=$ strongly disagree, $2=$ disagree, $3=$ neither agree nor disagree, 4 = agree, and 5 = strongly agree. We also wanted to use a response scale where each scale point had intrinsic meaning, such that for example, we could assess whether the participant believed the statement to be true. We calculated an overall problematic use score as the mean of retained items.

\subsubsection{Smartphone Use Scale}

The measure of smartphone use was taken from Van Deursen, Bolle, Hegner, and Kommers (2015). This included 6 items measuring habitual smartphone use, 7 items measuring what we label entertainment smartphone use (and which they label process use), and 5 items measuring social smartphone use. We felt that the label "process use" did not reflect the nature of the items which focus on using a smartphone to relax, read news, pass time, have a pleasant break, escape real-life, and be entertained. Items were rated on a 5-point scale: $1=$ strongly disagree, $2=$ disagree, 3 $=$ neither agree nor disagree, $4=$ agree, and $5=$ strongly agree . Scale scores were the mean of constituent items.

\subsubsection{Five Factor Model Personality}

The 300 item IPIP-NEO Inventory (Goldberg, 1999; Goldberg et al., 2006) was used to measure the Big 5 factors and 30 facets of the Five Factor Model (Costa \& McCrae, 2008). Items were rated on a 5 -point scale: $1=$ very inaccurate, $2=$ moderately inaccurate, $3=$ neither inaccurate nor accurate, $4=$ moderately 
accurate, $5=$ very accurate. The IPIP NEO has the advantage that it is in the public domain and as such, item level details can be provided in open science repositories. Correlations between corresponding NEO-PI-R and IPIP NEO scales have been found to range from .73 or .94 when corrected for reliability (Goldberg, 1999).

\subsubsection{HEXACO Personality}

The 200-item version of the HEXACO PI-R (Ashton et al., 2014; Lee \& Ashton, 2016) was used to measure personality factors and facets. The measure consists of 6 domain scales and 25 facet scales. Each domain scale consists of four facet scales, and there is one interstitial facet, altruism. Participants responded to items on a scale from $1=$ strongly disagree to $5=$ strongly agree. Scale scores were obtained as the mean of items after any necessary item reversal.

\subsection{Data Analytic Approach}

Data analysis was split into three sections. First, we assess the descriptive features and psychometric properties of the problematic and general smartphone use scales. Second, we examine bivariate correlations focusing particularly on the correlations of factors and facets of personality with smartphone use. When presenting facet-level correlations we present both zeroorder correlations (i.e. standard correlations) and residualized correlations. The residualized correlations are obtained by correlating the criteria with residualized facets, whereby variance that overlaps with broad traits is removed from the facet. For example, the residualized facet of FFM anxiety is obtained by taking the residual of a regression model predicting anxiety from the FFM domain scores. The resulting correlation is equivalent to the semi-partial correlation between facets and outcomes where facets are adjusted for overlap with corresponding domains (for discussion, see Anglim \& Grant, 2014, 2016; Lee \& Ashton, 2006). In general, given the large number of facets, an alpha of .001 was used to flag significant facet-level correlations. Third, a series of regression models are presented that compare the prediction of the four smartphone use variables (i.e., problematic, habitual, entertainment, and social) using different sets of personality predictors. Finally, a set of analyses examined the prediction of problematic use from a combination of smartphone use and personality. Overall prediction was assessed using the adjusted multiple correlation (i.e., the square root of adjusted rsquared). Using the adjustment provides for an unbiased estimate of the population relationship. This is particularly important when comparing models with different numbers of predictors (for discussion, see Anglim \& Grant, 2014, 2016). 


\section{Results}

\subsection{Preliminaries}

\subsubsection{Problematic Smartphone Use}

Table 1 presents the items measuring problematic smartphone use including item means, the percentage of participants who agreed or strongly agreed with the item and the correlation of the item with the final refined total score. In general, the percentage agreement with the items helps to convey the extent of problematic smartphone use. For example, $33 \%$ of participants reported feeling anxious when they have not checked phone messages, 34\% reported losing sleep due to time spent on their phone, $40 \%$ reported feeling lost without their phone, and 31\% reported decreased productivity as a direct result of time spent on their smartphone. Thus, overall, many participants indicated concerns about excessive use interfering with aspects of daily functioning.

Several items from the original scale were dropped due to the changing nature of smartphone use. Changes in cultural norms, such as increases in the saturation of smartphone ownership, changes to service provider billing practices, and changes to phone user interfaces were considered. Thus, we excluded items that did not intrinsically measure problematic smartphone use. For example, item 4 was simply an observation that other people own phones, and items 25 and 26 relate more to perceptions of other people. Item 11 pertains to making phone calls when feeling isolated. While feeling isolated may be problematic, this is not a symptom of mobile phone use, and using a mobile phone to chat or connect with others may be an adaptive way of dealing with feelings of isolation. In contrast, although arguments could be made both ways, item 2 ("I have used my mobile phone to make myself feel better when I was down"), while similar in nature to item 11, was not removed. Inclusion of Item 2 was partly based on the high item-total correlation (.57), compared to .37 for item 11 . In addition, item 2 is also likely to reflect a broader array of phone usage strategies including the more habitual and compulsive usage that is more representative of problematic usage. Other excluded items addressed secondary considerations (i.e., item 7 on billing issues, or item 25 on inconsiderate or disorganized use) or issues unrelated to the core phenomena (i.e., item 19 on aches and pains associated with mobile use, and item 15 about dreaming about mobile phone use). In addition, some excluded items had very high or low endorsement, and all but one had lower item-total correlations than most retained items (i.e., typically around .30 compared to the .60 range of retained items). 
Table 1

Descriptive Statistics for Problematic Mobile Phone Use

\begin{tabular}{|c|c|c|c|c|}
\hline Item Text & M & SD & $\%$ Agree & Item-total $\mathrm{r}$ \\
\hline \multicolumn{5}{|l|}{ Retained Items } \\
\hline 1. I can never spend enough time on my mobile phone. & 2.22 & 1.15 & 19 & .55 \\
\hline $\begin{array}{l}\text { 2. I have used my mobile phone to make myself feel better when I was feeling } \\
\text { down. }\end{array}$ & 3.36 & 1.19 & 60 & .57 \\
\hline $\begin{array}{l}\text { 3. I find myself occupied on my mobile phone when I should be doing other } \\
\text { things, and it causes problems }\end{array}$ & 3.35 & 1.20 & 54 & .71 \\
\hline 5. I have tried to hide from others how much time I spend on my mobile phone & 1.83 & 1.05 & 12 & .62 \\
\hline 6. I lose sleep due to the time I spend on my mobile phone. & 2.56 & 1.40 & 34 & 64 \\
\hline $\begin{array}{l}\text { 8. When out of range for some time, I become preoccupied with the thought of } \\
\text { missing a call }\end{array}$ & 1.92 & 1.12 & 15 & .57 \\
\hline $\begin{array}{l}\text { 9. Sometimes, when I am on the mobile phone and I am doing other things, I get } \\
\text { carried away with the conversation and I don't pay attention to what I am } \\
\text { doing }\end{array}$ & 2.79 & 1.22 & 40 & .59 \\
\hline 10. The time I spend on the mobile phone has increased over the last 12 months. & 2.65 & 1.23 & 28 & .55 \\
\hline 12. I have attempted to spend less time on my mobile phone but am unable to. & 2.28 & 1.05 & 15 & 65 \\
\hline 13. I find it difficult to switch off my mobile phone & 2.60 & 1.29 & 31 & .67 \\
\hline $\begin{array}{l}\text { 14. I feel anxious if I have not checked for messages or switched on my mobile } \\
\text { phone for some time }\end{array}$ & 2.61 & 1.26 & 33 & .66 \\
\hline 16. My friends and family complain about my use of the mobile phone & 1.74 & 1.00 & 9 & .54 \\
\hline $\begin{array}{l}\text { 18. My productivity has decreased as a direct result of the time I spend on the } \\
\text { mobile phone }\end{array}$ & 2.61 & 1.26 & 31 & .70 \\
\hline $\begin{array}{l}\text { 20. I find myself engaged on the mobile phone for longer periods of time than } \\
\text { intended }\end{array}$ & 3.39 & 1.25 & 65 & .69 \\
\hline $\begin{array}{l}\text { 21. There are times when I would rather use the mobile phone than deal with } \\
\text { other more pressing issues }\end{array}$ & 3.18 & 1.30 & 58 & .62 \\
\hline $\begin{array}{l}\text { 22. I am often late for appointments because I'm engaged on the mobile phone } \\
\text { when I shouldn't be }\end{array}$ & 1.52 & 0.84 & 6 & .59 \\
\hline $\begin{array}{l}\text { 23. I become irritable if I have to switch off my mobile phone for meetings, } \\
\text { dinner engagements, or at the movies }\end{array}$ & 1.52 & 0.84 & 5 & .57 \\
\hline 24. I have been told that I spend too much time on my mobile phone & 2.00 & 1.12 & 17 & .65 \\
\hline 27. I feel lost without my mobile phone & 2.83 & 1.28 & 40 & .63 \\
\hline \multicolumn{5}{|l|}{ Excluded Items } \\
\hline 4. All my friends own a mobile phone & 4.86 & 0.44 & 99 & -.03 \\
\hline 7. I have received mobile phone bills I could not afford to pay. & 1.57 & 1.03 & 11 & .36 \\
\hline 11. I have used my mobile phone to talk to others when I was feeling isolated & 3.77 & 1.15 & 77 & .34 \\
\hline 15. I have frequent dreams about the mobile phone & 1.18 & 0.48 & 1 & .37 \\
\hline $\begin{array}{l}\text { 17. If I don't have a mobile phone, my friends would find it hard to get in touch } \\
\text { with me. }\end{array}$ & 3.79 & 1.12 & 75 & .29 \\
\hline 19. I have aches and pains that are associated with my mobile phone use & 1.62 & 0.92 & 8 & .51 \\
\hline $\begin{array}{l}\text { 25. More than once I have been in trouble because my mobile phone has gone off } \\
\text { during a meeting, lecture, or in a theatre. }\end{array}$ & 1.39 & 0.69 & 3 & .38 \\
\hline 26. My friends don't like it when my mobile phone is switched off & 2.36 & 1.09 & 18 & .28 \\
\hline
\end{tabular}

Note. Response options ranged from $1=$ strongly disagree to $5=$ strongly agree. The percentage indicating agree or strongly agree was calculated. Item total correlation corresponds to the correlation with the overall measure of problematic phone use (excluded items were not used in calculating this scale). Item numbers correspond to those set out in Bianchi and Phillips (2005). 
In general, we adopted a one factor model for problematic smartphone use. The percentage of variance explained by the first six principal components in the 19 retained items was 38.7, 7.7, $6.9,5.0,4.6$, and 4.3. Thus, the first component was approximately five times larger than the second component. Because the scree plot provided slight evidence for three factors, we did inspect the factor loadings of a 3 factor solution using maximum likelihood extraction and promax rotation. The first factor tended to reflect compulsive use and negative consequence on sleep and productivity, the second factor tended to relate to anxiety when away from the phone, and the third factor had only two highly loading items concerned with friends complaining about smartphone use. In general, all three factors were highly correlated and seemed to reflect a common underlying dimension of problematic use. The lack of clear or compelling subscales justified our focus on a single problematic smartphone use scale.

\subsubsection{General Smartphone Use}

Descriptive statistics and factor loadings for smartphone use items are shown in Table 2. A maximum likelihood factor analysis with promax rotation showed that items loaded cleanly on the three theorized factors with no cross loadings above .30. With the exception of the escapism item, all items had relatively high levels of endorsement. For some items, it seems that the agreement is reaching a level where the use is a fundamental characteristic of owning a smartphone, in a society where smartphone use is nearly ubiquitous. 
Table 2

Descriptive Statistics and Factor Loadings for Smartphone Use Scale

\begin{tabular}{|c|c|c|c|c|c|c|}
\hline Item & $\mathrm{M}$ & $\mathrm{SD}$ & $\begin{array}{c}\% \\
\text { Agree }\end{array}$ & $\mathrm{I}$ & II & II \\
\hline \multicolumn{7}{|l|}{ Habitual Use } \\
\hline 1. Smartphone use is part of my daily routines & 4.39 & 0.68 & 95 & .59 & .04 & .07 \\
\hline 2. Checking my smartphone is becoming a habit & 4.21 & 0.93 & 89 & .92 & .03 & -.07 \\
\hline 3. I use my smartphone automatically & 4.16 & 0.91 & 86 & .91 & .01 & -.06 \\
\hline 4. It's a habit to use my smartphone & 4.13 & 0.97 & 85 & .97 & .01 & -.11 \\
\hline 5. My smartphone is a part of my life & 4.11 & 0.86 & 86 & .57 & .04 & .10 \\
\hline $\begin{array}{l}\text { 6. When I need to complete a certain task then the use of my } \\
\text { smartphone is an obvious choice }\end{array}$ & 3.73 & 1.06 & 70 & .42 & .13 & .07 \\
\hline \multicolumn{7}{|l|}{ Entertainment Use } \\
\hline 7. I use my smartphone in order to escape from real-life & 2.54 & 1.21 & 27 & .11 & .55 & -.10 \\
\hline 8. I use my smartphone in order to relax & 3.49 & 1.14 & 66 & -.09 & .77 & -.06 \\
\hline 9. I use my smartphone because it is entertaining. & 4.04 & 0.86 & 87 & .02 & .75 & .06 \\
\hline $\begin{array}{l}\text { 10. I use my smartphone because it informs me for things that } \\
\text { happen in everyday life }\end{array}$ & 3.95 & 0.98 & 83 & .14 & .31 & .15 \\
\hline $\begin{array}{l}\text { 11. I use my smartphone in order to stay up to date of the latest } \\
\text { news }\end{array}$ & 3.87 & 1.06 & 78 & -.06 & .46 & .27 \\
\hline 12. I use my smartphone because it helps me passing time & 3.83 & 1.07 & 75 & .05 & 68 & .03 \\
\hline $\begin{array}{l}\text { 13. I use my smartphone because it's a pleasant break from my } \\
\text { routines }\end{array}$ & 3.61 & 1.07 & 69 & .03 & .67 & -.01 \\
\hline \multicolumn{7}{|l|}{ Social Use } \\
\hline 14. I use my smartphone to interact with people & 4.37 & 0.76 & 94 & -.14 & .04 & .90 \\
\hline 15. I use my smartphone to maintain relationships & 4.07 & 0.97 & 82 & -.14 & .16 & .72 \\
\hline 16. I use my smartphone to call other people & 4.13 & 1.00 & 85 & .15 & -.25 & .45 \\
\hline 17. I use my smartphone to text message others & 4.53 & 0.62 & 97 & .12 & -.23 & .65 \\
\hline 18. I use my smartphone to contact people through social media & 4.30 & 1.02 & 91 & -.08 & .13 & .65 \\
\hline
\end{tabular}

Note. Response options ranged from $1=$ strongly disagree to $5=$ strongly agree. The percentage indicating agree or strongly agree was calculated. Factor loadings (Promax rotation) are shown in columns I, II, and II. Absolute factor loadings above .30 are bolded.

\subsubsection{Reliability Analysis}

Mean Cronbach's alpha for personality variables was .81 (range: .71 to .90) for the 30 FFM facets, .93 (range: .89 to .96) for the Big 5 FFM domains, .82 (range: .71 to .88) for the 25 HEXACO facets, and .89 (range: .80 to .94) for the 6 HEXACO domains. Cronbach's alpha for smartphone use variables was .91 for problematic use, .89 for habitual use, .83 for entertainment use, and .76 for social use. Alphas for each scale are presented in an online supplement.

\subsection{Correlations}

Table 3 presents the correlation between the outcome measures along with descriptive statistics (a complete correlation matrix of FFM facets, FFM domains, HEXACO facets, HEXACO domains, smartphone variables, and demographics, is available in an online supplement). Consistent with the emerging ubiquity of 
smartphones, the means for the three smartphone use scales were about a third of a standard deviation larger than the sample of Dutch adults in Van Deursen et al. (2015). The three measures of general smartphone use-habitual, social, and entertainmentwere strongly intercorrelated. Correlations with problematic smartphone use were larger for habitual use $(r=.53)$ and entertainment use $(r=.60)$ than for social use $(r=.27)$. Small correlations were observed with gender and age, whereby females and younger adults reported slightly more problematic and general use. Partial correlations controlling for age and gender are reported in the online supplement.

Table 3

Descriptive Statistics and Intercorrelations for Smartphone Use and Demographics

\begin{tabular}{lrllllll}
\hline Variable & M & SD & 1 & 2 & 3 & 4 & 5 \\
\hline 1. Problematic Use & 2.47 & 0.72 & & & & & \\
2. Habitual Use & 4.12 & 0.73 & .53 & & & & \\
3. Entertainment Use & 3.62 & 0.74 & .60 & .60 & & & \\
4. Social Use & 4.28 & 0.63 & .27 & .45 & .40 & & \\
5. Female & 0.79 & 0.41 & .14 & .13 & .16 & .12 & \\
6. Age & 24.42 & 7.14 & -.22 & -.24 & -.25 & -.16 & .05 \\
\hline
\end{tabular}

Note. Female (Male $=0 ;$ Female $=1)$. Absolute correlations equal to or larger than $.12, .13$, and .17 are statistically significant at .05 , .01 , and .001 respectively.

Table 4 presents the correlations of the broad Big 5 and HEXACO traits with smartphone use. Problematic smartphone use correlated most with Big 5 neuroticism (+) and conscientiousness (-). That said, small negative correlations were observed for Big 5 agreeableness and openness. In general, correlates with HEXACO traits showed some similarities, although correlations with emotionality (+) and conscientiousness (-) were somewhat smaller, and the correlations with openness (-) and, to a lesser extent, agreeableness (-) were somewhat larger. Honesty-humility showed a notable negative correlation with problematic use. Correlations with the general use scales showed distinct patterns. In particular, social smartphone use showed clear and substantial correlations with extraversion. In contrast, personality correlates with habitual and entertainment use largely mirrored the pattern of correlations observed for problematic use, albeit correlations tended to be smaller. 
Table 4

Correlations for HEXACO and Five Factor Model Broad Traits with Smartphone Use

\begin{tabular}{lcccc}
\hline Trait & $\begin{array}{c}\text { Problematic } \\
\text { Use }\end{array}$ & $\begin{array}{c}\text { Habitual } \\
\text { Use }\end{array}$ & $\begin{array}{c}\text { Entertainment } \\
\text { Use }\end{array}$ & $\begin{array}{c}\text { Social } \\
\text { Use }\end{array}$ \\
\hline FFM Neuroticism & $\mathbf{. 4 1}$ &. $\mathbf{2 2}$ &. $\mathbf{3 7}$ & .05 \\
FFM Extraversion & .00 & .16 & -.04 &. $\mathbf{3 7}$ \\
FFM Agreeableness & -.18 & -.10 & -.09 & .05 \\
FFM Conscientiousness & -.38 & -.14 & $\mathbf{- . 2 1}$ & -.06 \\
FFM Openness & -.15 & .05 & -.12 & .07 \\
& & & & \\
HEXACO Honesty-Humility & $\mathbf{- . 2 8}$ & $\mathbf{- . 2 7}$ & $\mathbf{- . 2 2}$ & -.13 \\
HEXACO Emotionality & $\mathbf{. 3 3}$ &. $\mathbf{2 7}$ & $\mathbf{. 3 1}$ & .17 \\
HEXACO Extraversion & -.11 & .06 & -.10 & .27 \\
HEXACO Agreeableness & $\mathbf{- . 2 1}$ & -.12 & $\mathbf{- . 2 1}$ & .03 \\
HEXACO Conscientiousness & $\mathbf{- . 2 7}$ & -.11 & -.16 & -.03 \\
HEXACO Openness & $\mathbf{- . 2 4}$ & -.12 & $\mathbf{- . 2 0}$ & -.11 \\
\hline
\end{tabular}

Note. Absolute correlations greater than or equal to .20 are bolded. Absolute correlations equal to or larger than .12, .13, and .17 are statistically significant at $.05, .01$, and .001 respectively. FFM = Five Factor Model.

Table 5 presents the facet-level correlations for the Five Factor Model facets. Zero-order correlations highlight at a descriptive-level which aspects of a broad trait are driving the domain-level correlations. For example, straightforwardness appears to show more prominent correlations with problematic use than other facets of agreeableness. Similarly, the facet of impulsiveness was the facet of neuroticism with the strongest correlation with problematic use. In the context of openness, the facets of actions, and especially ideas, showed notable negative correlations. Nonetheless, when residualized correlations were examined, only one facet-criteria combination was significant at .001 , suggesting that effect sizes related to incremental prediction by facets are likely to be small. 
Table 5

Zero-order and Residualized Correlations between Five Factor Model Personality Facets and Smartphone Use

\begin{tabular}{|c|c|c|c|c|c|c|c|c|}
\hline & \multicolumn{4}{|c|}{ Zero-order correlations } & \multicolumn{4}{|c|}{ Residualized Correlations } \\
\hline & PU & $\mathrm{HU}$ & EU & SU & PU & $\mathrm{HU}$ & EU & SU \\
\hline N1. Anxiety & .36 & .21 & .35 & .06 & .06 & .00 & .06 & -.05 \\
\hline N2. Angry hostility & .30 & .18 & .28 & .05 & -.11 & -.10 & -.07 & -.06 \\
\hline N3. Depression & .27 & .09 & .25 & -.02 & -.13 & -.12 & -.08 & .00 \\
\hline N4. Self-consciousness & .23 & .07 & .20 & -.12 & .11 & .11 & .01 & .04 \\
\hline N5. Impulsiveness & .41 & .32 & .35 & .21 & .06 & .11 & .09 & .07 \\
\hline N6. Vulnerability & .38 & .20 & .33 & .06 & .03 & .00 & .01 & -.02 \\
\hline E1. Warmth & -.04 & .09 & -.08 & .30 & .04 & .02 & .00 & .01 \\
\hline E2. Gregariousness & .09 & .13 & .01 & .35 & -.01 & -.09 & -.03 & .00 \\
\hline E3. Assertiveness & -.05 & .11 & -.05 & .24 & .01 & -.02 & .03 & -.02 \\
\hline E4. Activity & -.12 & .08 & -.12 & .11 & -.03 & .02 & -.11 & -.06 \\
\hline E5. Excitement seeking & .15 & .22 & .10 & .30 & .00 & .04 & .06 & .01 \\
\hline E6. Positive emotions & -.05 & .10 & -.04 & .30 & .00 & .02 & .04 & .06 \\
\hline O1. Fantasy & .05 & .07 & .05 & .09 & .11 & .02 & .12 & .10 \\
\hline O2. Aesthetics & -.09 & .06 & -.06 & .09 & -.01 & .01 & -.01 & .00 \\
\hline O3. Feelings & .10 & .22 & .15 & .16 & .03 & .10 & .07 & .01 \\
\hline O4. Actions & -.18 & -.04 & -.21 & .04 & -.03 & -.05 & -.08 & -.08 \\
\hline O5. Ideas & -.31 & -.12 & -.24 & -.08 & -.06 & -.09 & -.06 & -.06 \\
\hline O6. Values & -.11 & -.01 & -.12 & -.04 & -.05 & .01 & -.05 & .00 \\
\hline A1. Trust & -.08 & .03 & -.08 & .19 & .07 & .10 & .04 & .07 \\
\hline A2. Straightforwardness & -.29 & -.23 & -.11 & -.08 & -.16 & -.17 & -.05 & -.07 \\
\hline A3. Altruism & -.10 & .05 & -.04 & .23 & .07 & .08 & .07 & .09 \\
\hline A4. Compliance & -.14 & -.10 & -.13 & -.02 & .08 & .04 & -.02 & -.04 \\
\hline A5. Modesty & -.05 & -.14 & -.02 & -.14 & -.10 & -.08 & -.11 & -.03 \\
\hline A6. Tender-mindedness & -.07 & -.01 & .02 & .06 & .05 & .02 & .09 & .00 \\
\hline C1. Competence & -.34 & -.06 & -.21 & .02 & .00 & .08 & .05 & -.01 \\
\hline C2. Order & -.15 & -.05 & -.02 & -.08 & .03 & .04 & .04 & .00 \\
\hline C3. Dutifulness & -.35 & -.15 & -.20 & -.06 & -.09 & -.04 & -.07 & -.07 \\
\hline C4. Achievement striving & -.23 & -.02 & -.15 & .07 & .07 & .05 & -.02 & .04 \\
\hline C5. Self-discipline & -.33 & -.14 & -.19 & -.02 & -.02 & -.05 & .00 & .02 \\
\hline C6. Deliberation & -.32 & -.23 & -.18 & -.18 & -.01 & -.06 & -.01 & -.01 \\
\hline
\end{tabular}

Note. Absolute correlations greater than or equal to .17 are bolded. Absolute correlations equal to or larger than .12, .13, and .17 are statistically significant at $.05, .01$, and .001 respectively. $\mathrm{PU}=$ problematic smartphone use, $\mathrm{HU}=$ habitual smartphone use, $\mathrm{EU}=$ entertainment smartphone use, $\mathrm{SU}=$ social smartphone use.

Table 6 presents facet-level correlations for the HEXACO model. As with the Five Factor Model, there was limited evidence for strong incremental facet-level correlations, over and above domain level predictors, but the zero-order correlations helped to explain domain-level correlations. For example, the facet of sentimentality appeared less related to problematic use compared to anxiety and dependence. Aesthetic appreciation and inquisitiveness had more prominent correlations with problematic use than the other facets of openness. 
Table 6

Zero-order and Residualized Correlations between HEXACO

Personality Facets and Smartphone Use

\begin{tabular}{|c|c|c|c|c|c|c|c|c|}
\hline & \multicolumn{4}{|c|}{ Zero-order correlations } & \multicolumn{4}{|c|}{ Residualized Correlations } \\
\hline & $\mathrm{PU}$ & $\mathrm{HU}$ & EU & SU & PU & $\mathrm{HU}$ & EU & SU \\
\hline H1: Sincerity & -.25 & -.26 & -.21 & -.13 & .08 & -.01 & .06 & -.01 \\
\hline H2: Fairness & -.21 & -.17 & -.15 & -.04 & -.02 & -.04 & -.02 & .00 \\
\hline H3: Greed-Avoidance & -.20 & -.23 & -.21 & -.18 & .02 & -.02 & -.06 & -.06 \\
\hline H4: Modesty & -.19 & -.14 & -.10 & -.05 & -.07 & .07 & .03 & .08 \\
\hline E1: Fearfulness & .19 & .08 & .18 & -.09 & -.06 & -.11 & -.05 & -.18 \\
\hline E2: Anxiety & .26 & .20 & .26 & .08 & -.01 & .03 & .01 & .06 \\
\hline E3: Dependence & .32 & .27 & .26 & .27 & .07 & .04 & .03 & .07 \\
\hline E4: Sentimentality & .14 & .20 & .16 & .23 & -.01 & .05 & .02 & .07 \\
\hline X1: Social Self-Esteem & -.18 & .00 & -.14 & .16 & -.05 & .01 & -.01 & -.06 \\
\hline X2: Social Boldness & -.08 & .04 & -.08 & .19 & .01 & -.02 & .00 & -.04 \\
\hline X3: Sociability & .03 & .13 & .02 & .30 & .03 & .01 & .04 & .04 \\
\hline X4: Liveliness & -.14 & .02 & -.14 & .24 & .01 & .01 & -.03 & .06 \\
\hline A1: Forgiveness & -.11 & -.03 & -.17 & .08 & .04 & .06 & -.04 & .03 \\
\hline A2: Gentleness & -.17 & -.15 & -.15 & .03 & -.03 & -.10 & -.01 & .00 \\
\hline A3: Flexibility & -.12 & -.07 & -.08 & .04 & .02 & .04 & .10 & .03 \\
\hline A4: Patience & -.24 & -.14 & -.23 & -.04 & -.03 & .00 & -.04 & -.06 \\
\hline C1: Organization & -.17 & -.03 & -.03 & .03 & .02 & .08 & .12 & .10 \\
\hline C2: Diligence & -.23 & -.07 & -.15 & .00 & .01 & .00 & -.01 & -.04 \\
\hline C3: Perfectionism & -.08 & -.01 & -.09 & .00 & .10 & .05 & -.04 & .05 \\
\hline C4: Prudence & -.33 & -.24 & -.24 & -.15 & -.13 & -.14 & -.10 & -.13 \\
\hline O1: Aesthetic Appreciation & -.21 & -.10 & -.16 & -.07 & -.02 & .00 & .01 & .06 \\
\hline $\mathrm{O} 2$ : Inquisitiveness & -.28 & -.17 & -.21 & -.17 & -.08 & -.08 & -.03 & -.11 \\
\hline O3: Creativity & -.11 & -.06 & -.13 & -.01 & .10 & .01 & .04 & .07 \\
\hline O4: Unconventionality & -.13 & -.03 & -.14 & -.10 & .02 & .08 & -.01 & -.01 \\
\hline I: Altruism & -.01 & .06 & .02 & .08 & .08 & .12 & .09 & .03 \\
\hline
\end{tabular}

Note. Absolute correlations greater than or equal to .17 are bolded.

Absolute correlations equal to or larger than .12,.13, and .17 are statistically significant at $.05, .01$, and .001 respectively. $\mathrm{PU}=$ problematic smartphone use, $\mathrm{HU}=$ habitual smartphone use, $\mathrm{EU}=$ entertainment smartphone use, $\mathrm{SU}=$ social smartphone use.

\subsection{Regression Models}

A series of regression models were run to estimate the ability of various sets of personality traits to predict smartphone use (i.e. problematic, habitual, entertainment, social) from personality. Predictive validity was indexed using the adjusted multiple correlation. The adjustment means that it is an unbiased estimate of the corresponding population parameter, and thus provides a fair basis for comparing models with different numbers of predictors (for discussion, see Anglim \& Grant, 2014). Using adjusted multiple correlation (i.e., taking the square root of adjusted r-squared) allows direct comparison with the zero-order correlations. The main regression models were based on the following predictor sets: (a) the 5 FFM domains, (b) the 30 FFM facets, (c) the 6 HEXACO domains, and (d) the $25 \mathrm{HEXACO}$ 
facets. This enabled an assessment of whether facets provided incremental prediction over domains within both HEXACO and FFM frameworks, and whether HEXACO and FFM differed in predictive validity at domain and facet-levels. Finally, various composite models were estimated. In particular, honesty-humility was added to the Big 5 from the FFM to see whether honestyhumility improved prediction over and above the Big 5 alone. Then, composite models with (a) the 11 domains, and (b) the 55 facets of both models were estimated. These composite models assess whether HEXACO and FFM yield improved prediction over and above using only one personality framework.

Table 7 presents the adjusted multiple correlations for all of these models. Regression coefficients are presented in an online supplement. Consistent with problematic use being well predicted by a combination of high neuroticism and low conscientiousness, including all Big 5 domains resulted in a large adjusted multiple correlation (.53). Prediction by the six HEXACO domains was similar (.49). In general, the incremental prediction by facets over factors for both HEXACO and FFM models was fairly modest, whereby the increase was less than $10 \%$. Including both HEXACO and FFM domains significantly increased prediction of problematic use $(\mathrm{p}<.001)$. Adding honesty-humility to the Big 5 led to a small but significant increase in prediction $(\mathrm{p}=.01)$.

Table 7

Adjusted Multiple Correlations for Regression Models Predicting Smartphone Use from Different Sets of Personality Predictors

\begin{tabular}{|c|c|c|c|c|c|}
\hline Predictors & $\mathrm{k}$ & $\begin{array}{c}\text { Problematic } \\
\text { Use }\end{array}$ & $\begin{array}{c}\text { Habitual } \\
\text { Use }\end{array}$ & $\begin{array}{c}\text { Entertainment } \\
\text { Use } \\
\end{array}$ & $\begin{array}{c}\text { Social } \\
\text { Use }\end{array}$ \\
\hline \multicolumn{6}{|l|}{ Single Models } \\
\hline FFM Domains & 5 & .53 & .38 & .41 & .45 \\
\hline FFM Facets & 30 & .57 & .44 & .44 & .44 \\
\hline HEXACO Domains & 6 & .49 & .40 & .41 & .39 \\
\hline HEXACO Facets & 25 & .51 & .43 & .41 & .44 \\
\hline \multicolumn{6}{|l|}{ Composite Models } \\
\hline FFM Domains + Honesty-Humility & 6 & .54 & .41 & .43 & .45 \\
\hline HEXACO + FFM Domains & 11 & .56 & .44 & .45 & .47 \\
\hline HEXACO + FFM Facets & 55 & .58 & .45 & .44 & .47 \\
\hline
\end{tabular}

Note. $\mathrm{k}=$ number of predictors. FFM = Five Factor Model.

We also estimated a series of regression models predicting problematic use from the three general use scales and personality. The standardized betas using just general use were .28 for habitual, .45 for entertainment, and -.03 for social (adjusted multiple $\mathrm{r}=$ .64). When these general use factors were included in addition to 
age and gender, age and gender were no longer significant predictors of problematic use (adjusted multiple $r=.64$ ). A model with general use and the Big 5 showed that personality provided significant $(\mathrm{p}<.001)$ incremental prediction over and above general use with standardized betas of .25 for habitual, .35 for entertainment, -.05 for social, .20 for neuroticism, .16 for extraversion, - -10 for openness, -.03 for agreeableness and -.19 for conscientiousness (adjusted multiple $r=.70$ ). ). Similarly, a model with general use and the 6 HEXACO factors also showed that personality provided significant $(\mathrm{p}<.001)$ incremental prediction over and above general use with standardized betas of .24 for habitual, .37 for entertainment, -.03 for social, -.12 for honestyhumility, .14 for emotionality, -.03 for extraversion, .02 for agreeableness, -.12 for conscientiousness, and -.08 for openness (adjusted multiple $\mathrm{r}=.68$ ).

\section{Discussion}

The present study aimed to address a gap in the personality-smartphone literature by mapping personality factors and facets of two major personality frameworks (i.e., Five Factor Model and HEXACO) onto general and problematic smartphone use. Overall, the data showed relatively high levels of problematic smartphone use and very high levels of general use. Within the two personality frameworks under consideration, the FFM domains of neuroticism (+) and conscientiousness (-) had the strongest association with problematic smartphone use. Within the HEXACO framework, emotionality (+) had the strongest association with problematic smartphone use. The honestyhumility (-) domain had a moderate relationship with problematic smartphone use, as did agreeableness (-), conscientiousness (-), and openness (-). Interestingly, the extraversion domain in both models showed a negligible relationship with problematic smartphone use. In terms of general use, specific factors in both personality models had moderate correlations with general use. For example, extraversion (+) was moderately correlated with social use in both models. Neuroticism (FFM) and emotionality (HEXACO) were moderately associated with entertainment use, while habitual use was associated most strongly with the traits of honesty-humility (-) and emotionality (+), followed by neuroticism $(+)$. The addition of the facets in a regression model provided modest additional incremental prediction suggesting that the broad traits of neuroticism and conscientiousness do a reasonable job of capturing the disposition to engage in problematic smartphone use. Nonetheless, facet-level correlations provided a more nuanced understanding of which aspects of the broad traits were most relevant to predicting problematic smartphone use. 


\subsection{Personality and Smartphone Use}

Consistent with the emerging ubiquity of smartphones, the overall reported use of smartphones in all three categories (social, entertainment, and habitual) was very high. Social use was the most heavily endorsed type of use, however only slightly more than habitual use. In terms of specific personality factors, there were some traits that were associated with different types of general use more than others. For example, within the FFM, neuroticism demonstrated the strongest association with both habitual and entertainment use, while extraversion was most strongly associated with social use. The HEXACO model approximated the same pattern as the FFM, however the differential patterns were not as clear.

Neuroticism demonstrated the strongest relationship to problematic smartphone use in the present study. High neuroticism coupled with low self-esteem has been proposed as one 'pathway' to problematic smartphone use (Billieux, 2012). The theory is that individuals high in neuroticism tend to rely on their smartphones to obtain social and emotional reassurance from relationships (Kim, Seo, \& David, 2015). The need for frequent reassurance is thought to be based on the tendency of neurotic individuals to engage in maladaptive cognitions about the self or have insecure attachment styles (Billieux, 2012). In contrast however, Bianchi and Phillips (2005) found no relationship between neuroticism and problematic smartphone use. The explanation proposed by Bianchi and Phillips (2005) was that neuroticism is characterised by anxiety and moodiness, as well as a tendency to be overly emotional and highly reactive to stimuli. The loud, bright, and potentially irritating functions of a mobile phone may make it a stress-inducing device for highly neurotic individuals. However, given the highly customisable nature of smartphones in 2017, devices can be set up to have low-arousal features (e.g. silent mode, low screen illumination etc.) which may mean the effect proposed by Bianchi and Phillips (2005) no longer restricts the way that neurotic individuals engage with their devices.

Conscientiousness in both models also appears to be important in understanding the relationship between personality and problematic smartphone use. The negative relationship could be explained by the characteristics of conscientiousness (e.g. dutifulness, self-discipline, and deliberation) having a protective effect on users, insofar as high conscientiousness may reduce a person's tendency to habitually or mindlessly check their smartphone. Conscientious people are characterised by a willingness to delay short-term gratification in order to achieve socially sanctioned goals such as achieving in work and education, delivering on interpersonal commitments, and engaging in a 
healthy lifestyle. Many of the problematic forms of smartphone use are characterised by short-term and illusory benefits, such as the notifications and recognition obtained on social media, or the constant stream of light entertainment and news.

Interestingly, the present study found that extraversion in both models was relatively uncorrelated with problematic smartphone use. This differs from some earlier studies where extraversion was associated with problematic use (Bianchi \& Phillips, 2005; Billieux, 2012; Takao, 2014). Past studies have proposed that the highly social nature of extraverts may lead them to engage in excessive use of social media platforms that may then lead to habitual and problematic use. It may be that this relationship is changing as the evolution of mobile phones changes from a simple voice and text communications device to the internet-enabled mini-computer that is modern-day smartphones. Results of the current study showed that extraversion was related to social smartphone usage, however habitual and entertainment use were more related to problematic use. It may be that while extraverts are more inclined to engage in online social behaviours, their positive personality may mitigate the potential for social smartphone use to become worrisome or obsessive.

The HEXACO model showed similarities and differences with the Five Factor model. Overall, the prediction of problematic use at the level of broad traits was very similar (adjusted multiple $r$ of .53 for FFM and .49 for HEXACO). In general, the pattern of correlations was fairly similar, although emotionality and conscientiousness were important predictors in the HEXACO model, they did not stand out as much as neuroticism and conscientiousness did in the FFM. In general, while emotionality is most similar to neuroticism, it does draw on some broader concepts. In particular, sentimentality (e.g., crying in sad movies, empathizing when friends are sad) moves the broad trait of emotionality a little away from the core of negative affect captured in FFM neuroticism.

Results also showed that honesty-humility had moderate negative correlation with problematic use. Although the correlation was not as large as often seen when predicting criteria related to deception and immoral behavior, it may be that people who are greedy, immodest, and insincere-characteristics of low honestyhumility - also place excessive value on some of the short-term rewards provided by obsessive smartphone and social media use. Past research has also shown links between honesty-humility and self-control (De Vries \& Van Gelder, 2013). A low honestyhumility individual may also find that manipulation of others via direct message or pointed social media posts is less confronting 
than face-to-face communication, thereby increasing the likelihood of smartphone use or misuse.

In general, narrow personality traits provided additional insight into the nature of dispositional tendencies leading to problematic smartphone use. Impulsiveness $(+)$, vulnerability $(+)$, prudence $(-)$, and dependence $(+)$ were the facets that demonstrated the strongest correlations with problematic smartphone use. On face value, being more impulsive, more vulnerable, and more dependent are traits that could easily be attributed to a tendency to develop problematic smartphone use. Prudence is a conscientiousness facet in the HEXACO model that refers to a tendency to deliberate carefully, limit impulses, and be cautious when considering consequences to actions. These characteristics also have good face validity in terms of understanding their role in predicting problematic smartphone use. A number of other facets had clear face-value relationships with problematic smartphone use (e.g. anxiety (+), competence (-), self-discipline (-), and inquisitiveness (-), Tables $5 \& 6)$. Although the Big 5 are an adequate set of predictors, the addition of the facets allows for a more fine-grained analysis of the specific personality characteristics that seem to be important in understanding problematic smartphone use.

Interestingly, while we found that females and younger adults were somewhat more likely to report problematic smartphone usage, personality was a stronger predictor of problematic smartphone use than these demographic variables. It is interesting to contrast this finding with Kim, Briley and Ocepek (2015) who found that demographic characteristics were better predictors of smartphone use than personality. While their large representative sample offers an excellent representation of the population from which it was drawn, there are several key differences between their study and the present study. First, they used the Ten Item Personality Inventory (TIPI, Gosling, Rentfrow, $\&$ Swann, 2003). The use of only two-items per Big 5 factor leads to substantially reduced reliability and validity relative to longform measures of personality, such as the IPIP NEO or HEXACO Personality Inventory. This in turn substantially attenuates correlations between personality and criteria such as smartphone usage. Second, Kim et al (2015) investigated demographic and personality correlates with the use of smartphone functions (e.g. social networking, listening to music), not problematic smartphone use. It seems likely that personality traits like conscientiousness and neuroticism will be more relevant to excessive, compulsive, and maladaptive use than binary indicators of usage of a particular smartphone application. Finally, in Kim et al's (2015) study only $43.8 \%$ of participants used smartphones, whereas in our study 
$98.7 \%$ of participants used smartphones. In the three or more years since Kim et al (2015) collected usage data, smartphone uptake and ownership has increased substantially. Thus, in many countries, almost all mobile phones are smartphones, and demographic factors initially related to early adoption may be disappearing. As the functionality and addictive potential of smartphones increases and ownership reaches saturation point, the influence of demographic factors on problematic smartphone usage may become more nuanced.

\subsection{Limitations}

Several limitations should be noted. First, the participants were a convenience sample of university students who were more likely to be female and tended to be younger than the general population. We performed supplementary analyses examining the interaction effects of age or gender with personality (i.e., the 6 HEXACO and 5 FFM domains) on problematic smartphone use and found no significant interactions at the .01 level. Nonetheless, smartphone usage is a culturally and technologically bounded phenomena that varies across social groups and countries and changes over time. Thus, future studies could seek to replicate and extend this work by examining relationships between personality and problematic smartphone usage in different target populations.

Second, while the IPIP NEO and smartphone scales were administered in the same session, the HEXACO personality inventory was administered a few weeks later. The delay was intended to reduce participant fatigue. While personality traits are highly stable over time, the delay may also lead to slightly reduced correlations with the second inventory compared to what would be obtained were both personality and outcome measures administered in the same session. Similarly, HEXACO correlations may also have been slightly more attenuated due to reliability because the HEXACO measure used 200 items ( 8 per facet, 32 per domain) whereas the IPIP used 300 items (10 per facet, 60 per domain).

Third, the study relied on self-reported use. One way to mitigate this effect would be to collect usage data from the device itself (e.g., Shin \& Dey, 2013; Stachl et al., 2017). A second approach could be to obtain both self and other-ratings, in order to determine whether an individual's perceptions of their use mirrors the perceptions of a close other.

Finally, the study highlights the need for measures and definitions of problematic smartphone use to be updated over time. In particular, endorsement of some smartphone usage items appears to be so ubiquitous that they are no longer measuring individual differences. For example, $97 \%$ of participants "[use their] smartphone to text message others" and 95\% report 
"smartphone use [being] part of [their] daily routines". Given that smartphones have partially filled the role of technologies such as landline telephones, postal mail, computers, banks, and supermarkets, it is perhaps not surprising that many people access their smartphones daily. They may be doing many of the same daily activities of five or ten years ago, just from a single mobile device rather than from multiple fixed-location resources. Perhaps a more pragmatic measure of smartphone use would begin by assuming that smartphones are a part of most people's daily routines, and instead measure time spent on the smartphone each day, rather than simply whether or not a person uses their smartphone on a daily basis. In a similar sense, the norms regarding what is adaptive smartphone use and what is excessive or inappropriate are changing.

\subsection{Conclusion}

The current study aimed to provide the most comprehensive mapping of personality to problematic smartphone use to date. We used two comprehensive and long-form measures of personality to provide valid and reliable measurement of broad and narrow traits based on two major hierarchical frameworks of personality. This was combined with a sample size sufficiently large enough to enable the subtle examination of facet-level correlates. In general, smartphones are both useful and addictive devices, and are likely to become more so as functionality increases and social norms adapt to their increased integration into daily life. Recognising the traits and characteristics of individuals who may be more prone to developing problematic smartphone use is a step in the direction of understanding the mechanisms that underlie this behaviour.

\section{References}

Anglim, J., Bozic, S., Little, J., \& Lievens, F. (2018). Response Distortion on Personality Tests in Applicants: Comparing High-Stakes to Low-Stakes Medical Settings. Advances in Health Science Education, 23, 311-321.

Anglim, J., \& Grant, S. L. (2014). Incremental Criterion Prediction of Personality Facets over Factors: Obtaining Unbiased Estimates and Confidence Intervals. Journal of Research in Personality, 53, 148-157.

Anglim, J., \& Grant, S. L. (2016). Predicting psychological and subjective wellbeing from personality: Incremental prediction from 30 facets over the Big 5. Journal of Happiness studies, 17(1), 59-80.

Anglim, J., Knowles, E. R. V., Dunlop, P. D., \& Marty, A. (2017). HEXACO Personality and Schwartz's Personal Values: A Facet-Level Analysis. Journal of Research in Personality, 68, 23-31.

Anglim, J., Morse, G., De Vries, R. E., MacCann, C., \& Marty, A. (2017). Comparing Job Applicants to Non-Applicants Using an Item-Level Bifactor Model on the HEXACO Personality Inventory. European Journal of Personality, 31, 669-684.

Ashton, M., Lee, K., \& de Vries, R. E. (2014). The HEXACO HonestyHumility, Agreeableness, and Emotionality factors: a review of 
research and theory. Personality and Social Psychology Review, 18, 139-152.

Bianchi, A., \& Phillips, J. G. (2005). Psychological predictors of problem mobile phone use. CyberPsychology \& Behavior, 8(1), 39-51.

Billieux, J. (2012). Problematic use of the mobile phone: a literature review and a pathways model. Current Psychiatry Reviews, 8(4), 299-307.

Billieux, J., Philippot, P., Schmid, C., Maurage, P., De Mol, J., \& Van der Linden, M. (2015). Is Dysfunctional Use of the Mobile Phone a Behavioural Addiction? Confronting Symptom-Based Versus ProcessBased Approaches. Clinical Psychology \& Psychotherapy, 22(5), 460468.

Butt, S., \& Phillips, J. G. (2008). Personality and self reported mobile phone use. Computers in Human Behavior, 24(2), 346-360.

Christensen, M. A., Bettencourt, L., Kaye, L., Moturu, S. T., Nguyen, K. T., Olgin, J. E., . . Marcus, G. M. (2016). Direct Measurements of Smartphone Screen-Time: Relationships with Demographics and Sleep. PLoS ONE, 11(11), e0165331-e0165331.

Collier, R. (2016). Mental health in the smartphone era. CMAJ: Canadian Medical Association Journal = Journal De L'association Medicale Canadienne, 188(16), 1141-1142.

Costa, P., \& McCrae, R. (1992). Normal personality assessment in clinical practice: The NEO Personality Inventory. Psychological assessment, 4(1), 5.

Costa, P., \& McCrae, R. (2008). The Revised NEO Personality Inventory (NEOPI-R). The SAGE handbook of personality theory and assessment, 2, 179-198.

David, P., Kim, J., Brickman, J. S., Ran, W., \& Curtis, C. M. (2014). Mobile phone distraction while studying. New Media \& Society, 17(10), 16611679.

De Vries, R., \& Van Gelder, J.-L. (2013). Tales of two self-control scales: Relations with Five-Factor and HEXACO traits. Personality and Individual Differences, 54(6), 756-760.

Deloitte. (2016a). 2016 Global Mobile Consumer Survey: US Edition Retrieved from

Deloitte. (2016b). Mobile Consumer Survey 2016 The Australian Cut Retrieved from Sydney:

Demirci, K., Akgönül, M., \& Akpinar, A. (2015). Relationship of smartphone use severity with sleep quality, depression, and anxiety in university students. Journal of Behavioral Addictions, 4(2), 85-92.

Dunlop, P. D., Lee, K., Ashton, M. C., Butcher, S. B., \& Dykstra, A. (2015). Please accept my sincere and humble apologies: The HEXACO model of personality and the proclivity to apologize. Personality and Individual Differences, 79, 140-145.

Elhai, J. D., Dvorak, R. D., Levine, J. C., \& Hall, B. J. (2017). Problematic smartphone use: A conceptual overview and systematic review of relations with anxiety and depression psychopathology. Journal of Affective Disorders, 207, 251-259.

Elhai, J. D., Levine, J. C., Dvorak, R. D., \& Hall, B. J. (2016). Fear of missing out, need for touch, anxiety and depression are related to problematic smartphone use. Computers in Human Behavior, 63, 509-516.

Ezoe, S., Toda, M., \& Yoshimura, K. (2009). Relationships of personality and lifestyle with mobile phone dependence among female nursing students. Soc Behav Pers Int J, 37.

Goldberg, L. R. (1999). A broad-bandwidth, public domain, personality inventory measuring the lower-level facets of several five-factor models. Personality Psychology in Europe, 7, 7-28. 
Goldberg, L. R., Johnson, J. A., Eber, H. W., Hogan, R., Ashton, M., Cloninger, C. R., \& Gough, H. G. (2006). The international personality item pool and the future of public-domain personality measures. Journal of Research in Personality, 40(1), 84-96.

Gosling, S. D., Rentfrow, P. J., \& Swann, W. B. (2003). A very brief measure of the Big-Five personality domains. Journal of Research in Personality, 37(6), 504-528.

Hawi, N. S., \& Samaha, M. (2016). To excel or not to excel: Strong evidence on the adverse effect of smartphone addiction on academic performance. Computers \& Education, 98, 81-89.

Hormes, J. M., Kearns, B., \& Timko, C. A. (2014). Craving Facebook? Behavioral addiction to online social networking and its association with emotion regulation deficits. Addiction, 109(12), 2079-2088.

Horwood, S., \& Anglim, J. (2017). A Critical Analysis of the Assumptions of Type D Personality: Comparing Prediction of Health-Related Variables with the Five Factor Model. Personality and Individual Differences, $117,172-176$.

Horwood, S., Anglim, J., \& Tooley, G. (2015). Type D Personality and the FiveFactor Model: A Facet-Level Analysis. Personality and Individual Differences, 83, 50-54.

Hussain, Z., Griffiths, M. D., \& Sheffield, D. (2017). An investigation into problematic smartphone use: The role of narcissism, anxiety, and personality factors. Journal of Behavioral Addictions, 6(3), 378-386.

Ishii, K. (2011). Examining the adverse effects of mobile phone use among Japanese adolescents. Keio Communication Review, 33, 69-83.

Kardaras, N. (2016). Glow Kids: How screen addiction is hijacking our kids and how to break the habit. New York: St Martin's Press.

Kay, R., Benzimra, D., \& Li, J. (2017). Exploring Factors That Influence Technology-Based Distractions in Bring Your Own Device Classrooms. Journal of Educational Computing Research, 55(7), 974995.

Kayiş, A. R., Satici, S. A., Yilmaz, M. F., Şimşek, D., Ceyhan, E., \& Bakioğlu, F. (2016). Big five-personality trait and internet addiction: A metaanalytic review. Computers in Human Behavior, 63, 35-40.

Kim, J., Seo, M., \& David, P. (2015). Alleviating depression only to become problematic mobile phone users: Can face-to-face communication be the antidote? Computers in Human Behavior, 51(Part A), 440-447.

Kim, Y., Briley, D. A., \& Ocepek, M. G. (2015). Differential innovation of smartphone and application use by sociodemographics and personality. Computers in Human Behavior, 44, 141-147.

Kim, Y., Jeong, J. E., Cho, H., Jung, D. J., Kwak, M., Rho, M. J., . . Choi, I. Y. (2016). Personality factors predicting smartphone addiction predisposition: Behavioral Inhibition and Activation Systems, Impulsivity, and Self-Control. PLoS ONE, 11(8).

Lee, K., \& Ashton, M. C. (2004). Psychometric properties of the HEXACO personality inventory. Multivariate behavioral research, 39(2), 329358.

Lee, K., \& Ashton, M. C. (2005). Psychopathy, Machiavellianism, and narcissism in the Five-Factor Model and the HEXACO model of personality structure. Personality and Individual Differences, 38(7), 1571-1582.

Lee, K., \& Ashton, M. C. (2006). Further assessment of the HEXACO Personality Inventory: two new facet scales and an observer report form. Psychological assessment, 18(2), 182-191.

Lee, K., \& Ashton, M. C. (2016). Psychometric properties of the HEXACO-100. Assessment, 1073191116659134. 
Lee, K., Ashton, M. C., Morrison, D. L., Cordery, J., \& Dunlop, P. D. (2008). Predicting integrity with the HEXACO personality model: Use of selfand observer reports. Journal of Occupational and Organizational Psychology, 81, 147-167.

Lee, K., Gizzarone, M., \& Ashton, M. C. (2003). Personality and the likelihood to sexually harass. Sex Roles, 49, 59-69.

Lee, U., Lee, J., Ko, M., Lee, C., Kim, Y., Yang, S., . . Song, J. (2014). Hooked on smartphones: An exploratory study on smartphone overuse among college students. Paper presented at the Conference on Human Factors in Computing Systems - Proceedings.

Lee, Y.-K., Chang, C.-T., Lin, Y., \& Cheng, Z.-H. (2014). The dark side of smartphone usage: Psychological traits, compulsive behavior and technostress. Computers in Human Behavior, 31, 373-383.

Lemola, S., Perkinson-Gloor, N., Brand, S., Dewald-Kaufmann, J., \& Grob, A. (2015). Adolescents' Electronic Media Use at Night, Sleep Disturbance, and Depressive Symptoms in the Smartphone Age. Journal of Youth \& Adolescence, 44(2), 405-418.

Lepp, A., Barkley, J. E., Sanders, G. J., Rebold, M., \& Gates, P. (2013). The relationship between cell phone use, physical and sedentary activity, and cardiorespiratory fitness in a sample of U.S. college students. International Journal of Behavioral Nutrition and Physical Activity, $10(1), 79$.

Lepp, A., Li, J., Barkley, J. E., \& Salehi-Esfahani, S. (2015). Exploring the relationships between college students' cell phone use, personality and leisure. Computers in Human Behavior, 43, 210-219.

Long, J., Liu, T. Q., Liao, Y. H., Qi, C., He, H. Y., Chen, S. B., \& Billieux, J. (2016). Prevalence and correlates of problematic smartphone use in a large random sample of Chinese undergraduates. BMC Psychiatry, 16(1).

Matar Boumosleh, J., \& Jaalouk, D. (2017). Depression, anxiety, and smartphone addiction in university students- A cross sectional study. PLoS ONE, 12(8), e0182239-e0182239.

Otero-López, J. M., \& Villardefrancos Pol, E. (2013). Compulsive buying and the Five Factor Model of personality: A facet analysis. Personality and Individual Differences, 55, 585-590.

Owens, J. (2014). Insufficient Sleep in Adolescents and Young Adults: An Update on Causes and Consequences. Pediatrics, 134(3), e921.

Paunonen, S. V., \& Jackson, D. N. (2000). What is beyond the big five? Plenty! Journal Of Personality, 68(5), 821-835.

Pearson, C., \& Hussain, Z. (2015). Smartphone use, addiction, narcissism, and personality: A mixed methods investigation. International Journal of Cyber Behavior, Psychology and Learning, 5(1), 17-32.

Phillips, J. G., Butt, S., \& Blaszczynski, A. (2006). Personality and self-reported use of mobile phones for games. Cyberpsychology and Behavior, 9(6), 753-758.

Roberts, J. A., Pullig, C., \& Manolis, C. (2015). I need my smartphone: A hierarchical model of personality and cell-phone addiction. Personality and Individual Differences, 79, 13-19.

Schweizer, A., Berchtold, A., Barrense-Dias, Y., Akre, C., \& Suris, J.-C. (2017). Adolescents with a smartphone sleep less than their peers. European Journal Of Pediatrics, 176(1), 131-136.

Shaw, H., Ellis, D. A., Kendrick, L.-R., Ziegler, F., \& Wiseman, R. (2016). Predicting Smartphone Operating System from Personality and Individual Differences. CyberPsychology, Behavior \& Social Networking, 19(12), 727-732. 
Shin, C., \& Dey, A. K. (2013). Automatically detecting problematic use of smartphones. Paper presented at the UbiComp 2013 - Proceedings of the 2013 ACM International Joint Conference on Pervasive and Ubiquitous Computing.

Stachl, C., Hilbert, S., Au, J. Q., Buschek, D., De Luca, A., Bischl, B., . . Bühner, M. (2017). Personality Traits Predict Smartphone Usage. European Journal of Personality.

Sween, M., Ceschi, A., Tommasi, F., Sartori, R., \& Weller, J. (2017). Who is a Distracted Driver? Associations between Mobile Phone Use while Driving, Domain - Specific Risk Taking, and Personality. Risk analysis, 37, 2119-2131.

Takao, M. (2014). Problematic mobile phone use and big-five personality domains. Indian Journal of Community Medicine, 39(2), 111-113.

Takao, M., Takahashi, S., \& Kitamura, M. (2009). Addictive personality and problematic mobile phone use. CyberPsychology \& Behavior, 12, 501507.

Tindell, D. R., \& Bohlander, R. W. (2012). The Use and Abuse of Cell Phones and Text Messaging in the Classroom: A Survey of College Students. College Teaching, 60(1), 1-9.

Toda, M., Ezoe, S., Mure, K., \& Takeshita, T. (2016). Relationship of smartphone dependence to general health status and personality traits among university students. Open Journal of Preventive Medicine, 6(10), 215-221.

Van Deursen, A., Bolle, C. L., Hegner, S. M., \& Kommers, P. A. (2015). Modeling habitual and addictive smartphone behavior: The role of smartphone usage types, emotional intelligence, social stress, selfregulation, age, and gender. Computers in Human Behavior, 45, 411420.

Wang, J.-L., Wang, H.-Z., Gaskin, J., \& Wang, L.-H. (2015). The role of stress and motivation in problematic smartphone use among college students. Computers in Human Behavior, 53, 181-188.

Wentworth, D. K., \& Middleton, J. H. (2014). Technology use and academic performance. Computers \& Education, 78, 306-311.

Yen, J., Ko, C., Yen, C., Wu, H., \& Yang, M. (2007). The comorbid psychiatric symptoms of Internet addiction: attention deficit and hyperactivity disorder (ADHD), depression, social phobia, and hostility. Journal of Adolescent Health, 41(1), 93-98.

Yıldız, M. A. (2017). Emotion regulation strategies as predictors of internet addiction and smartphone addiction in adolescents. Journal of Educational Sciences \& Psychology, 7(1), 66-78. 